\title{
Ultraefficient Voltage Doubler Based on a GaN Resonant Switched-Capacitor Converter
}

\author{
Miroslav Vasici ${ }^{(0)}$, Member, IEEE, Diego Serrano ${ }^{(\bullet}$, Victor Toral, Pedro Alou ${ }^{(0}$. Member; IEEE, \\ Jesus A. Olive ${ }^{\circledR}$, Member, IEEE, and Jose A. Cobos ${ }^{\circledR}$, Senior Member, IEEE
}

\begin{abstract}
In this paper, we present a highly efficient and compact voltage doubler based on a resonant suitched-capacitor converter implemented with GaN FETs. Two possible approaches for its implementation are analyzed and compared. In the first approach, the resonant inductor is placed in series with a resonant capacitor, conducting a sinusoidal current, while in the second, it is placed in series with the input source, conducting rectified sinusoidal current Both resonant converters have the same voltage gain, and although the change in the position of the resonant inductor is, at the first glance, of minor importance, the analysis and results show that it has huge impact on the capability to achieve zero-voltage switching (ZVS) transitions at low output power. The experimental results clearly show that at low loads when the resonant inductor is in series with the resonant capacitor, the suitching frequency can be significantly higher than the resonant frequency and that it is, practically, impossible to achieve ZVS transitions, forcing the implementation of cycle skipping. The prototype implemented for the experiments can provide up to 4.5-kW' losing between 20 and $22 \mathrm{~W}$. In the case of light load ( $500 \mathrm{~W}$ '), the power losses were only 2-3 W'. Its power density is higher than $65 \mathrm{~kW} / \mathrm{dm}^{3}$. The same resonant converter was tested with Si CoolMOS devices as well and the impact of the semiconductor technology on the overall power losses was verified. Due to higher $C_{\text {oss }}$ capacitance, the $\mathrm{Si}$-based converter has $\mathbf{4 0 \%}$ higher power losses at full power than its GaN-based counterpart. The components of the $\mathrm{GaN}$-based converter occupy only $65 \mathrm{~cm}^{3}$, which opens a possibility to obtain a design with extremely high power density.
\end{abstract}

Index Tenns-Resonant converters, switched capacitors, zerovoltage suitching (ZVS).

\section{INTRODUCTION}

$\mathbf{P}$ OWER conversion systems can generally be grouped into single-stage architectures and multistage architectures. Although the multistage architectures are usually costly and more complex, they are used in the cases when the singlestage approach cannot achieve high performance, while meeting requirements such as wide operating range, high power density, and efficiency. In a multistage approach each part of the system is optimally designed to address one part of the desired requirements. Recently, this approach has been applied to achieve highly compact and dense solutions, decreasing the size of the passive components [1]-[3]. What is common in the recently presented works, it is that the first stage is based on a switched capacitor converter (SCC) and, normally, this stage is usually employed to provide multiple voltage levels in an efficient way. Using multilevel converters, the size and power losses of magnetic components, inductors and transformers, the most bulky components, in dc dc converters, are decreased.

SCCs have been used as a simple and low cost dc dc converter in low-power applications [4]-[6]. These converters normally operate with fixed voltage gains but with extremely high efficiency. In addition, due to the lack of magnetic components, they occupy very small volumes obtaining ultrahigh power densities. Nevertheless, one of the important issues of these converters is the switching losses, especially if the load power reaches kilowatt levels. In order to avoid high switching losses, a resonant inductor is combined with the "flying" capacitor, producing resonance, and by correct timing of the transistor control signals, it is possible to obtain soft-switching transitions, zero voltage switching (ZVS). As a consequence, the resonant switched-capacitor converter (RSCC) seems to be more suitable for a high-power application than the SCC [7]-[10]. The resonant inductor is smaller than the inductor employed in classical pulsewidth modulation converters because it does not have to store the energy. A voltage divider/doubler based on a switching capacitor is a very well known solution [11], [12], and, in this paper, we present a compact and ultraefficient voltage doubler and discuss two possible implementations, depending on the position of the resonant inductor. The resonant inductor can be placed in series with the resonant capacitor conducting a sinusoidal current, or it can be placed between the input source and middle point of the controllable switches conducting rectified resonant current (dc current) (see Figs. 1 and 2). Both resonant converters have the same voltage gain, and although the change in the position of the resonant inductor is, at the first glance, of minor importance, the analysis and results show that it has huge impact on the capability to achieve ZVS transitions at low output power and on the inductordesign and powerlosses. The analyzed converters were designed for input voltage between 300 and $500 \mathrm{~V}$, providing up to $4.5 \mathrm{~kW}$ of output power. In order to obtain high efficiency, GaN FETs have been used and the influence of the transistor technology on the converter design will be discussed. This fixed gain power converter can be used in applications such as photovoltaic boost converter where a high-voltage gain and high efficiency in combination 


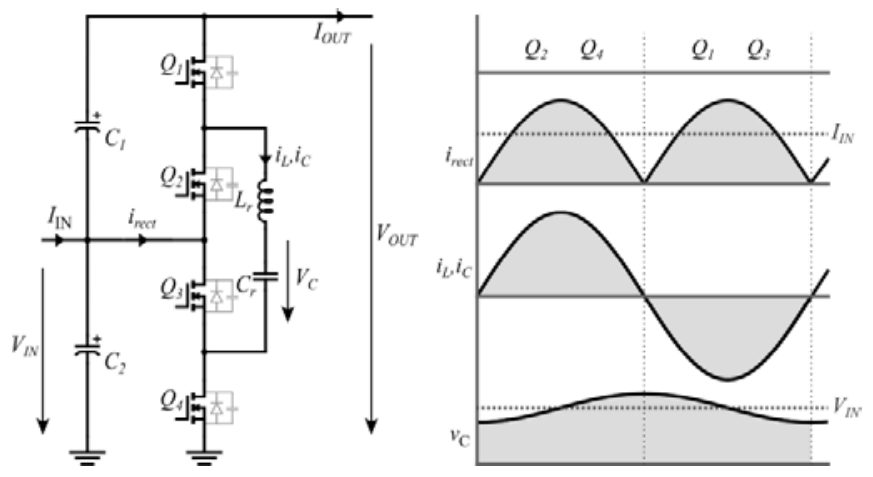

Fig. 1. RSCC with an ac resonant inductor.

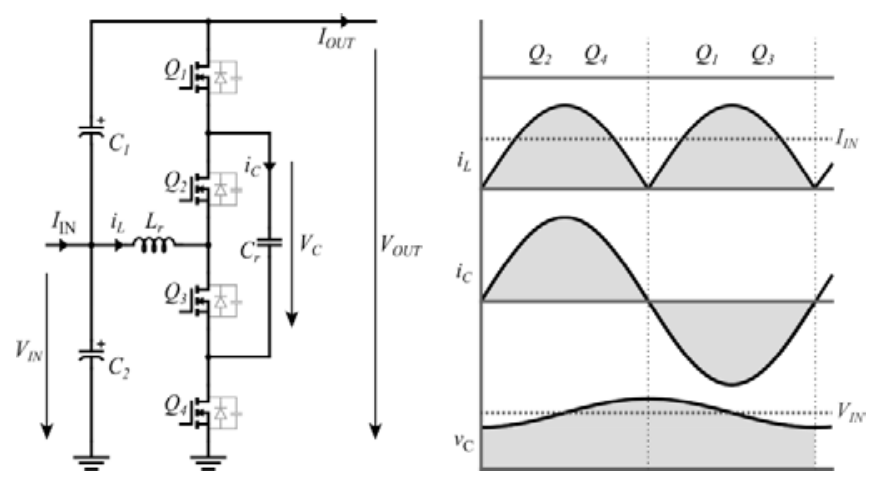

Fig. 2. RSCC with a de resonant inductor.

with low specific weight is needed. Hybrid converters using this RSCC in high-power application are previously presented and explained in [13] and [14], while the analyzed RSCC is presented in [15] as a part of a hybrid boost rectifier.

Schaef and Stauth [16] and Schaef et al. [17] propose slightly modified operation of this topology in order to obtain regulated output voltage, accepting decrement of the efficiency. They vary the duration of some sections of the switching period that are used in this paper to obtain ZVS transitions, in order to control the output voltage in the certain range of input voltages and loads. In this way, the obtained average value of the voltage of the resonant tank can be higher than $V_{\text {out }} / 2$ (boost mode) or lower than $V_{\text {out }} / 2$ (buck mode) and, thus, the ratio between the input and output voltage is higher or lower than 2 (the nominal gain). In addition, the interleaving technique of the basic resonant cell stage with common dc side inductor is proposed in [17]. However, these details are behind the scope of this paper and will be considered as a subject of the future work. In this work, operation with equal voltage distribution among the dc bus capacitors and constant boosting factor of 2 will be considered.

In [18] and [19], solutions on similar resonant switchedcapacitor architectures were presented to provide a variable gain using binary SCC and zero-current switching. Nevertheless, at input voltage levels that are relatively high $(350 \mathrm{~V})$, ZVS is of the outmost importance as it will be shown later.

\section{OPERATING PRinciple of THE Resonant SWITCHED-CAPACITOR CONVERTER}

Simplified schematic of the RSCC that operates with a voltage gain of two and its most important waveforms is shown in Figs. 1 and 2. In the case when the resonant inductor is placed in series with the resonant capacitor, its current is purely sinusoidal due to the resonance and due to the fact that the average value of the capacitor current has to be zero. In this case, we will denominate as a converter with ac resonant inductor. The duty cycle of the converter is, approximately, 50\%. During the first half of the switching cycle, the resonant network is connected to capacitor $C_{2}$ and the load current completely flows through $C_{1}$. During this period of time, the resonant capacitor is charged from the input source. In the second half of the switching cycle, the resonant network is connected in parallel to $C_{1}$ and its role is to compensate the charge taken from $C_{1}$ in the first half of the switching period and to provide the load current. Therefore, we can write the following equations for the $C_{1}$ current:

$$
\begin{aligned}
& i_{C 1}=I_{o} t \epsilon\left[0, \frac{T}{2}\right] \\
& i_{C 1}=I_{o}-I_{R} \sin (\omega t) t \epsilon\left[\frac{T}{2}, T\right]
\end{aligned}
$$

where $I_{0}$ is the load current and $I_{\mathrm{R}}$ is the amplitude of the resonant current. As the net charge of this capacitor must be equal to zero over one switching period by averaging its current, it is obtained that

$$
I_{R}=\pi I_{o}
$$

If we analyze the current $I_{\text {rect }}$ that flows between the middle point of $C_{1}$ and $C_{2}$ and the middle point of $\mathrm{Q}_{2}$ and $\mathrm{Q}_{3}$, we can conclude that this current has the waveform of the rectified resonant current. Therefore, if we place the resonant inductor between these points, the resonance will be maintained and the circuit will provide output voltage two times higher than the input just like in the previous case. In this case, we will denominate as a converter with $\mathrm{dc}$ resonant inductor. Although there is no difference how these two circuits operate, the inductor displacement produces three important changes.

The first one is obvious and it is related with the current through the resonant inductor. The rectified sine wave current has major part of its spectrum in the dc part; therefore, it is possible to exploit low dc resistance of the windings and decrease the power losses in the windings. Nevertheless, the core losses are influenced by the frequency and amplitude of the magnetic flux change. The excursion of the magnetic flux is just one half in comparison with the original design but double the resonant frequency. This might provoke higher core losses in the design. The second important change is the voltage level that turned-off switches must sustain. In the case of the ac inductor resonant circuit, the switch voltage is clamped to the input voltage. Nevertheless, in the case of dc inductor resonant circuit the switches must block voltage of the flying (resonant) capacitor and it is higher than the input voltage as this voltage has certain voltage ripple that depends 


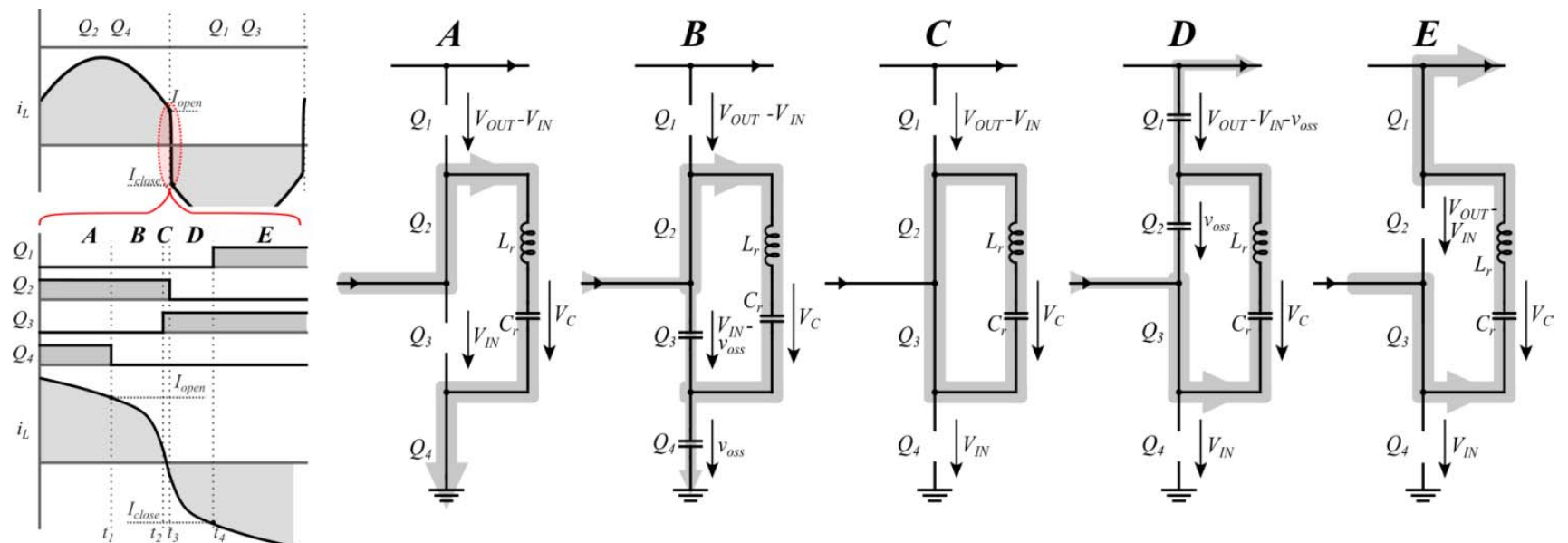

Fig. 3. ZVS transition in the case of the RSCC with an ac resonant inductor.
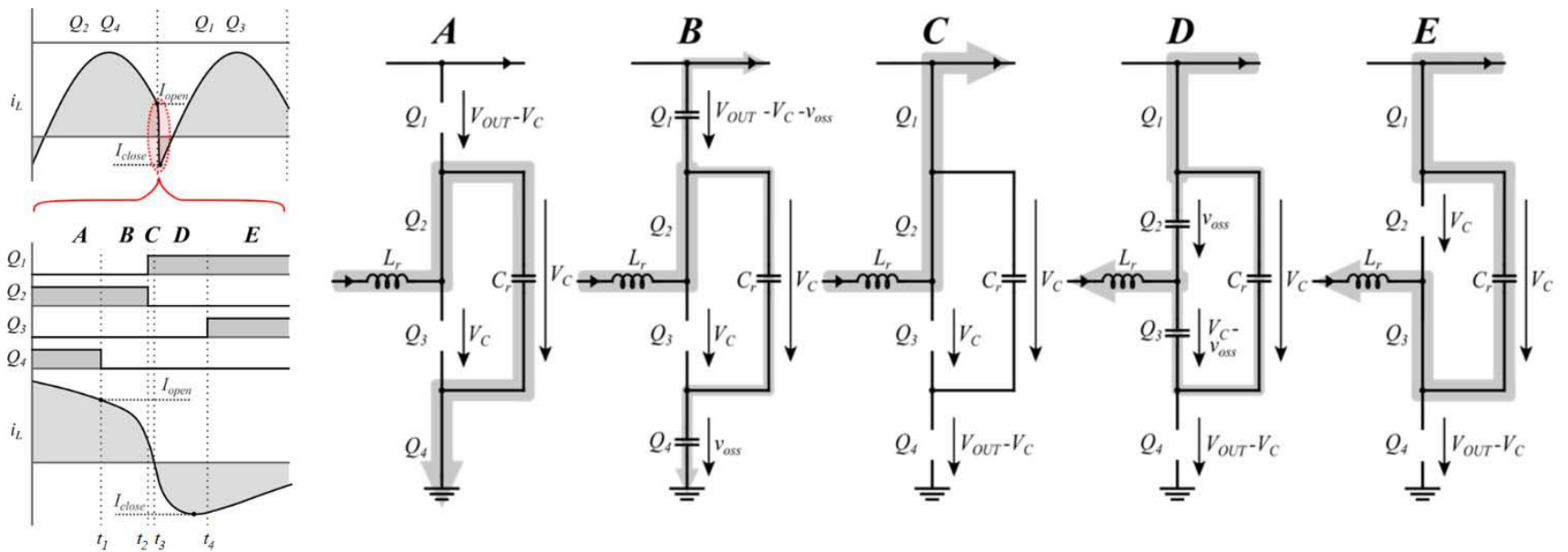

Fig. 4. ZVS transition in the case of the RSCC with a dc resonant inductor.

on the output power and value of the resonant capacitor. This might condition the selection of the device if the voltage ripple is not negligible. The last important change is regarding the control signals sent to the switches in order to obtain the ZVS transitions, like it is shown in Figs. 3 and 4.

During this transition, two transistors must be turned off $\left(\mathrm{Q}_{2}\right.$ and $\left.\mathrm{Q}_{4}\right)$ and two transistors must be turned on $\left(\mathrm{Q}_{1}\right.$ and $\left.\mathrm{Q}_{3}\right)$. In case of both analyzed converters, the ZVS transition starts when $\mathrm{Q}_{4}$ turned off. It must be turned off with sufficiently high current level, $I_{\text {open }}$, in order to have enough energy in the resonant inductor to accomplish soft transition. The first ZVS transition occurs between $t_{1}$ and $t_{2}$ in Figs. 3 and 4. During this time, the resonant current interacts with transistor's $C_{\text {oss }}$ capacitances, discharging $C_{\text {oss }}$ of $\mathrm{Q}_{1}$ and charging $C_{\text {oss }}$ of $\mathrm{Q}_{4}$ in the case of dc resonant inductor and discharging $C_{\text {oss }}$ of $\mathrm{Q}_{3}$ and charging $C_{\text {oss }}$ of $\mathrm{Q}_{4}$ in the case of ac resonant inductor.

Afterward, during very short period of time, from $t_{2}$ to $t_{3}$, there will be two transistors conducting in both converters and actually this time period can be skipped, although inherently will exist due to driver delays during very short moment. The second ZVS transition occurs from $t_{3}$ to $t_{4}$. During this time period, the resonant current is rising and becoming more negative and the energy of the resonant inductor is rising, facilitating ZVS transition fully charging $C_{\mathrm{oss}}$ of $\mathrm{Q}_{2}$ and fully discharging $C_{\text {oss }}$ of $\mathrm{Q}_{3}$. Until $t_{4}$, the resonant current behaves in the same way in both analyzed converters and in both cases it will approximately reach the value of

$$
I_{\text {close }}=-\sqrt{\frac{2 V_{\text {in }} V_{0} C_{0} N}{L}}
$$

where $N$ is the number of the transistors in parallel, $L$ is the value of the resonant inductor, and $C_{0}$ is the value of $C_{\mathrm{oss}}$ capacitance at low-voltage levels.

After $t_{4}$, the resonant current is negative and in the case of the converter with ac resonant inductor it will decrease, while in the case of the converter with dc resonant inductor it will start increasing. This is the key difference between the analyzed converters as it has a major impact on the switching frequency. The difference in the switching frequency comes from a fact that in the case of the dc resonant inductor the resonant current must change its sign, reach value of $I_{\mathrm{R} 1} \approx \pi I_{0}$ and then fall to $I_{\mathrm{open}}$. This lasts very close to a half of the resonant period. On the other hand, with the ac resonant inductor, the resonant current decreases to $-I_{\mathrm{R} 2} \approx-\pi I_{0}$ and then rises to $-I_{\mathrm{open}}$, like it is shown in Fig. 3. In this case, the switching period is obviously shorter than the resonant 


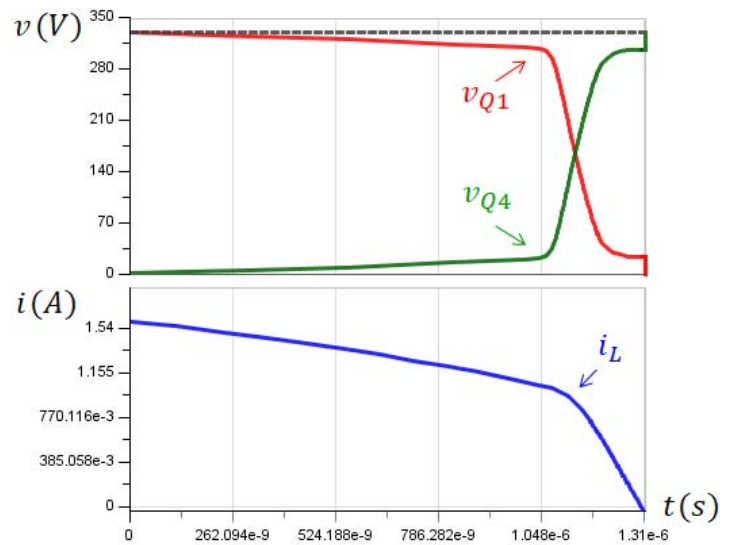

Fig. 5. Partial ZVS transition from $t_{1}$ to $t_{2}$.

and it will depend on the value of the load current, i.e., the difference between $-I_{\mathrm{R}}$ and $I_{\min }$. This difference is shorter as the load current is decreased and in some extreme cases when $I_{\min }$ decreases to $-I_{\mathrm{R}}$ the switching frequency can be several times higher than the resonant frequency of the converter. This means that in the case of the converter with ac resonant inductor the switching frequency will be load dependent, i.e., the lower the load, the higher is the switching frequency, which leads to high switching losses at light load which is exactly what should be avoided.

It is important to notice that as there are two transistors that must be closed during the described transient and that we need to guarantee that two soft switching events will occur. The first occurs at the end of period B and the second at the end of period D. In order to obtain the first soft switched event, it is of paramount importance to open $\mathrm{Q}_{4}$ with enough amount of resonant current in the resonant inductor, as the ZVS transition must finish before the resonant current reaches zero. On the other hand, the second transition is not a critical one, as the resonant current starts to rise at the beginning of period D. Consequently, period $\mathrm{B}$ will condition the design of the resonant inductor and selection of the resonant current that will be used for the ZVS transition and must be analyzed in detail as the transistor technology ( $\mathrm{Si}$ or $\mathrm{GaN})$ will have an important impact on the design.

In this paper, the analyzed converter can operate up to $1000 \mathrm{~V}$ and for this reason the employed devices are rated for $650 \mathrm{~V}$. Si CoolMOS or GaN FETs could be used, and the behavior of the converter during the ZVS transition will be different due to their $C_{\text {oss }}$. For both technologies, especially for CoolMOS, this capacitance could be modeled with a piecewise linear characteristic and roughly approximated with only two values. At low voltages, the capacitance has relatively high values, while at high voltages, the capacitance is very low [20], [21]. Due to this nonlinearity, the resonant current does not have a sinusoidal shape between $t_{1}$ and $t_{2}$, and Fig. 5 shows more details regarding this transition. Using the aforementioned simplification of transistor output capacitance, $C_{\text {oss }}$ is modeled as:

$$
C_{\mathrm{oss}}= \begin{cases}C_{0}, & V_{\mathrm{ds}}<V_{0} \\ C_{1}, & V_{\mathrm{ds}}>V_{0}\end{cases}
$$
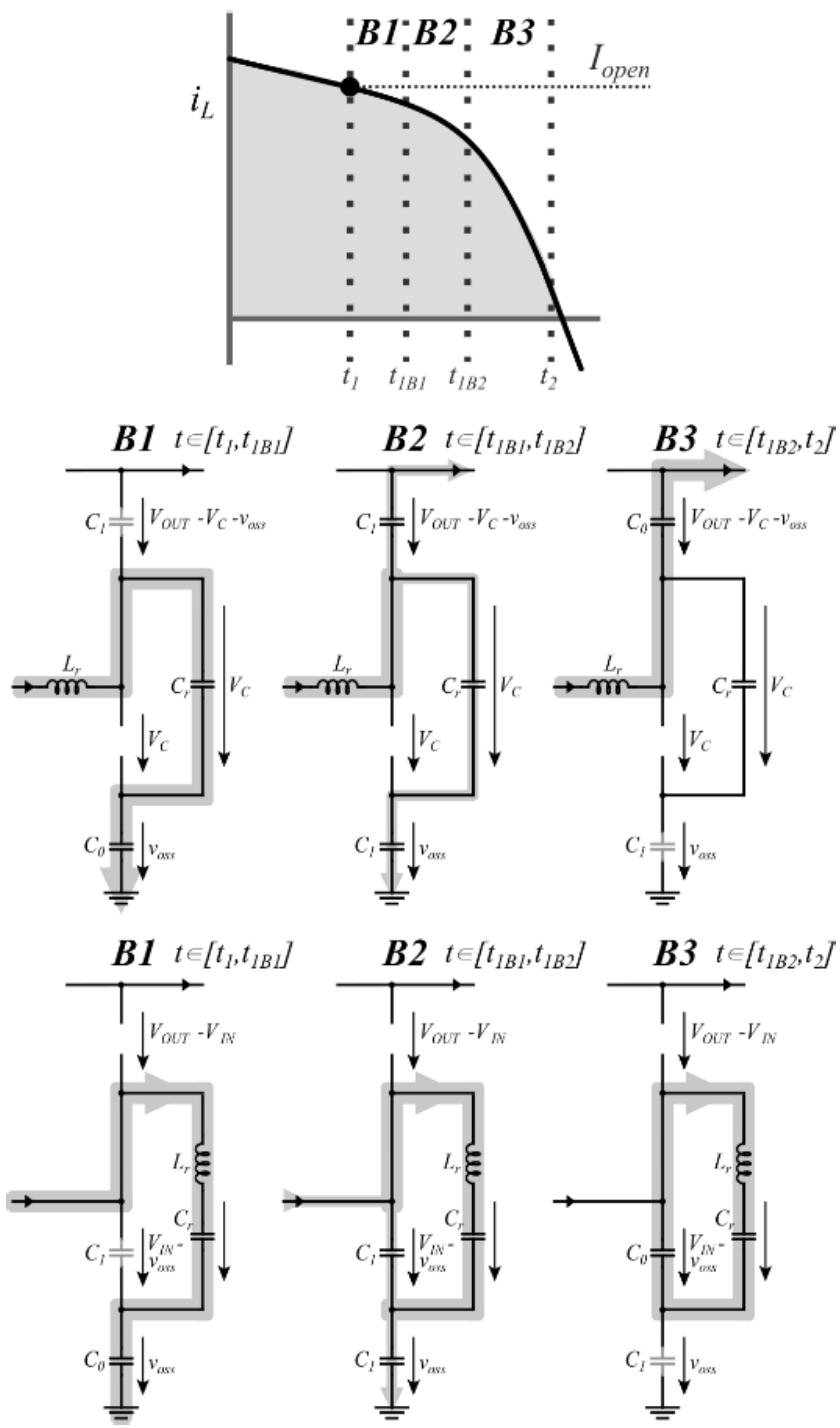

Fig. 6. Detailed ZVS transition from $t_{1}$ to $t_{2}$.

where $C_{1} \ll C_{0}$. For example, in the case of IPB65R045C7, rough parameters of this model are:

$$
C_{0}=30 \mathrm{nF}, \quad C_{1}=90 \mathrm{pF}, \quad V_{0}=20 \mathrm{~V}
$$

while in the case of GS66516T the values are

$$
C_{0}=1 \mathrm{nF}, \quad C_{1}=150 \mathrm{pF}, V_{0}=50 \mathrm{~V} .
$$

Using this approximation for transistor $C_{\text {oss }}$, the time interval B could be divided into three shorter intervals (B1, B2, and B3) like in Fig. 6. As it can be seen, in the case of the ac resonant inductor, at the very beginning of the first subinterval, $\mathrm{Q}_{3}$ withstands the complete input voltage, while $\mathrm{Q}_{4}$ has zero volts between its drain and source. This means that $\mathrm{Q}_{3}$ should be modeled as a small capacitance $C_{1}$, while $\mathrm{Q}_{4}$ as a big capacitance $C_{0}$.

As $C_{1} \ll C_{0}$, practically, only $C_{0}$ will have influence on the circuit behavior, drain-source voltages will change relatively slowly until the moment when VDS4 is charged up to $V_{0}$. At this moment, both transistors could be modeled with $C_{1}$ and at that moment drain-source voltages start to change rapidly 


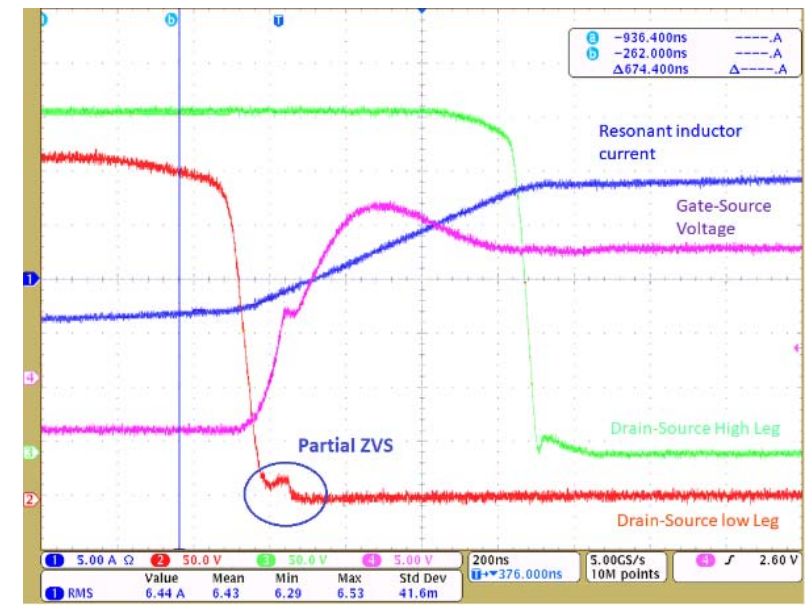

Fig. 7. Partial ZVS transition in the case of Si CoolMOS transistors due to high low-voltage $C_{\text {oss }}$.

together with the inductor current. This model is valid until the moment when $\mathrm{Q}_{3} C_{\text {oss }}$ is discharged up to $V_{0}$. From that moment, the circuit model is changed accordingly, $C$ oss $3=$ $C_{0}$ and $C_{\mathrm{oss} 4}=C 1$. In other words, $C_{\mathrm{oss} 4}$ can be neglected, and the simplified equivalent circuit is like the one in Fig. 6, interval B3. This last subinterval B3 rises the problem as the resonant inductor must discharge $C_{\mathrm{oss} 3}$ and, at the same time it charges the resonant capacitor, as it can be seen in Fig. 6. During this process, the inductor sustains high reverse voltage and its current decreases quickly. Ideally, the resonant current and voltage across $C_{\mathrm{oss} 3}$ reach zero at $t_{2}$. Nevertheless, in order to have both conditions fulfilled, the energy in the resonant inductor at $t_{1}$ has to be considerably high, especially if there are several transistors in parallel per switch. If we assume that the resonant current is changed, practically, linearly from $I_{\mathrm{L}}\left(t=t_{1 \mathrm{~B} 2}\right)$ to zero and that the resonant capacitor is charged to the input voltage, then, the following relationship can be obtained:

$$
2 V_{\text {in }} C_{0} V_{0}=L I_{\mathrm{L}}^{2}\left(t=t_{1 B 2}\right)
$$

This simple equation clearly shows the importance of $C_{0}$ for this subinterval and the semiconductor technology impact on ZVS conditions. If we compare IPB65R045C7 and GS66516T, it is clear that due to $C_{0}$ difference of 30 times it is easier to obtain ZVS with GaN than with $\mathrm{Si}$. In order to obtain ZVS with Si CoolMOS the level of the energy in the resonant inductor must be increased to a sufficient level. This can be obtained either by increasing the level of the resonant current in the moment when the ZVS transition is started or by increasing the value of the inductor. The first approach leads to higher turn-off losses, while the second one to an unacceptably bulky inductor. Therefore, in the case of Si CoolMOS, a tradeoff must be made by performing a "partial ZVS," i.e., the switch is closed when the voltage across $C_{\text {oss }}$ reaches $20-40 \mathrm{~V}$ [22]. In other words, at $t_{2}$ only the resonant current will reach zero and $C_{\mathrm{oss} 3}$ charge will be discharged through the channel of $\mathrm{Q}_{3}$ producing additional power losses (see Fig. 7), while in the case of $\mathrm{GaN}$, this is not the case and full ZVS can be reached without any problems (see Fig. 8). It is interesting to notice that in both experiments the same resonant inductor was used

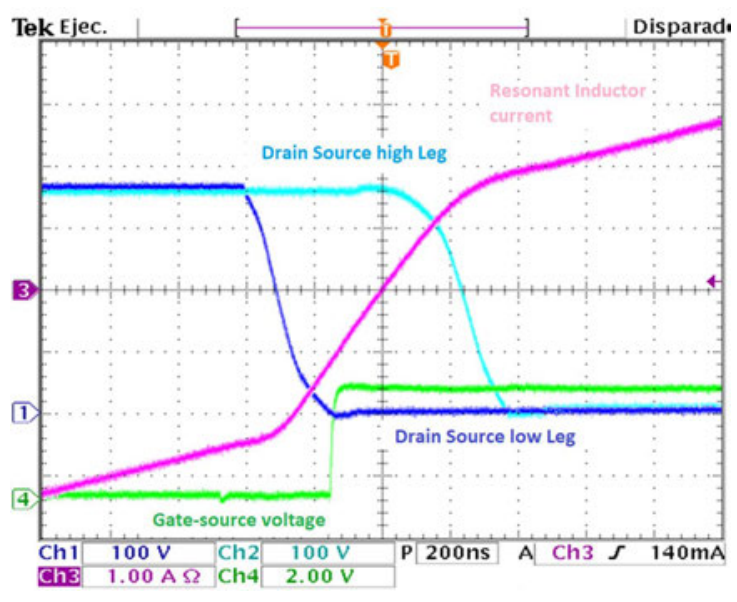

Fig. 8. Full ZVS transition in the case of GaN FETs.

and that $I_{\text {open }}$ in the case of CoolMOS was $4 \mathrm{~A}$ and in the case of GaN FET only 2 A. If the partial ZVS had not been applied, an inductance that is, approximately, 30 times bigger $(1.6 \mathrm{mH})$ or an $I_{\text {open }}$ of $11 \mathrm{~A}$ should have been employed in the case of CoolMOS which would lead to unacceptable size or power losses.

As it can be seen from previous explanations, we would like to have ZVS transitions at all load conditions and this will have influence on the switching frequency of the analyzed voltage doubler. In the case of ac resonant circuit, the resonant current is changing from $-I_{\text {close }}$ to $I_{\text {open }}$ (both currents are approximately 2 A with GaN FETs) and at low loads, the peak current is comparable with these two values. In other words, the inductor resonant current is losing its sinusoidal shape becomes more trapezoidal and the switching frequency is significantly higher than the resonant. This becomes an important problem, as all the power loss mechanisms dependent on the switching frequency become very dominant at low loads, significantly penalizing the converter efficiency. In addition, the converter cannot operate correctly at no-load conditions. In the case of dc resonant circuit, this problem is not as dominant as in the case of ac resonant circuit, as the resonant current starts with slightly negative value $\left(I_{\text {close }} \approx-2 \mathrm{~A}\right)$ and ends with slightly positive value $\left(I_{\text {open }}=2 \mathrm{~A}\right)$.

The switching frequency is, practically, maintained through complete load range and, even, it is operating at no-load conditions (as it will be shown later in the experimental results). Fig. 9 illustrates how the resonant current is behaving at different loads in the analyzed resonant circuits

One possible solution to tackle the problem of the switching frequency at light loads in the case of the ac resonant inductor could be pulse skipping modulation, as shown in Fig. 10. The main idea is to apply periods of time during which the resonant inductor will not be used to transfer the charge to the flying capacitor and to the load. As the total amount of charge that must be given to the load must remain the same as before, the amplitude of the resonant current will increase when the resonant current is activated. In that way, at low loads the peak of the resonant current is increased, and the switching frequency is, once again, similar to the resonant frequency and high-power losses could be avoided. 

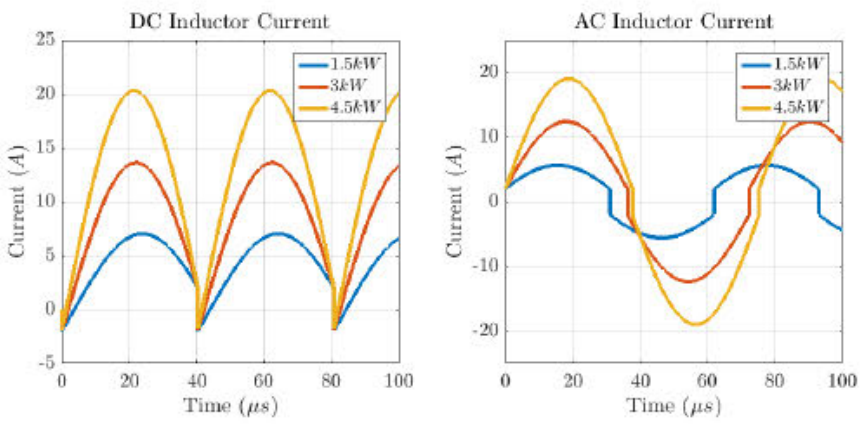

Fig. 9. Comparison of RSCC resonant currents in the case of different loads- $\mathrm{dc}$ and ac resonant inductor design.

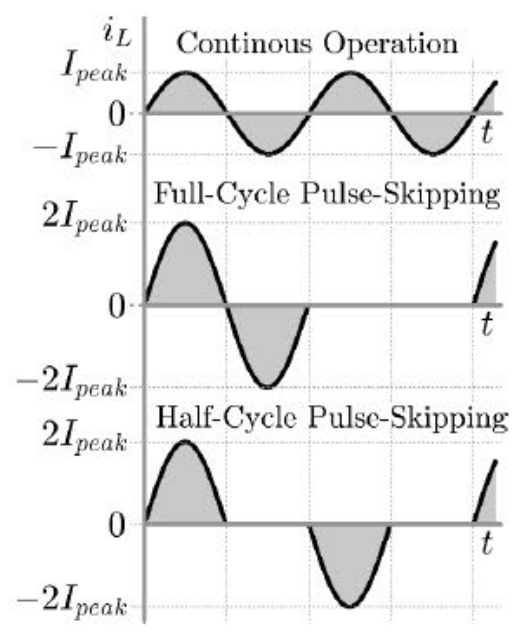

Fig. 10. Comparison of the resonant inductor current in the case of continuous and pulse skipping modulation.

Nevertheless, this technique brings some other problems (ZVS loss and high-frequency oscillations) and the power savings thanks to this technique will be detaily explained and quantified in Section IV. It is important to notice that the same peak of the resonant current can be obtained with different pulse skipping strategies, denominated as half-cycle and full-cycle pulse skipping, like it is shown in Fig. 10.

\section{Design, ANALysis, AND COMParison OF RESONANT CONVERTERS}

In order to perform a systematic analysis of both approaches, a design obtained by exploring the very limits of converter's efficiency and size was implemented. The design has been performed by modeling inductor, capacitor, and semiconductor losses and obtaining the Pareto front for both approaches ac and dc resonant inductors. Semiconductor losses are calculated by the model presented in [23]. Inductor core losses are calculated by improved general Steinmetz equation that is discussed in [24] and implemented in [25]. For winding losses calculations skin and proximity effects are considered [26]-[28]. DC bus and resonant capacitor losses are calculated using the data sheet information regarding their equivalent series resistance (ESR) at different operating points. The nonlinear behavior of multilayer ceramic chip capacitors was taken into account in order to correctly estimate the volume they occupy.
TABLE I

ELECTRICAL SPECIFICATIONS OF THE ANALYZED RESONANT CONVERTER

\begin{tabular}{|l|c|}
\hline Input voltage range & $300 \mathrm{~V}-500 \mathrm{~V}$ \\
\hline Maximum output power & $4.5 \mathrm{~kW}$ \\
\hline Nominal input voltage & $350 \mathrm{~V}$ \\
\hline Nominal output power & $1.5 \mathrm{~kW}$ \\
\hline
\end{tabular}

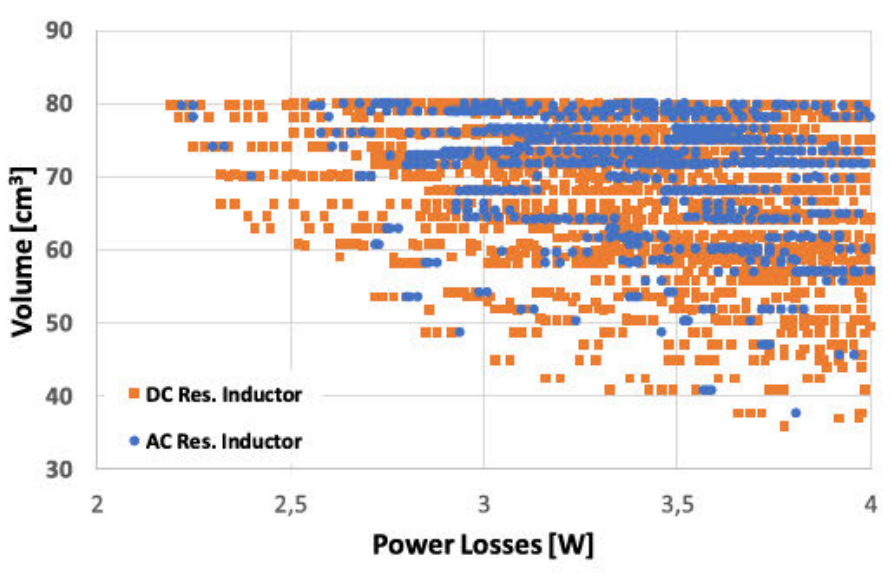

Fig. 11. Pareto front of the analyzed RSCCs.

The electrical specifications are presented in Table I and obtained Pareto fronts in Fig. 11. The optimization process was implemented in MATLAB scripts in which different inductor designs (cores, wire type, and number of turns) and semiconductor configuration were varied. Solutions that could not withstand full-load conditions were excluded, and Fig. 11 presents possible solutions that can operate correctly under the given specifications. The Pareto front in Fig. 11 shows power losses for the nominal conditions, but each design can survive the worst case. For this analysis, GaN GS66516T FETs have been considered for the switching device. Fig. 11 shows the volume that is equal to a simple sum of all component volumes.

As it can be seen, the approach based on a dc resonant inductor gives solutions that are slightly more compact or more efficient in comparison with the approach based on an ac inductor. For example, with dc and ac resonant inductor it is possible to obtain designs that occupy around $50 \mathrm{~cm}^{3}$ with power losses around $3 \mathrm{~W}$ at nominal conditions. Due to great performance of GaN FETs, it must be stressed that the difference between the designs is not as high as in the case when $\mathrm{Si}$ CoolMOS are employed [22] and the main reason for this is low $C_{\text {oss }}$ capacitance of the employed $\mathrm{GaN}$ transistors. As explained earlier, in the case of the ac inductance, the switching frequency is load dependent and it is correlated with the transistor's parasitic capacitance. When $\mathrm{Si}$ CoolMOS is used with the ac inductor resonant converter, the switching frequency at nominal load $(1.5 \mathrm{~kW})$ is three times higher than the resonant [22] and this is not the case with the GaN technology. With GaN FETs, this effect occurs at significantly lower power levels, around $500 \mathrm{~W}$ as it will be seen later in the experimental results. In the case of the ac resonant inductor, the load-dependent switching frequency 
TABLE II

DESIGN DETAILS OF THE IMPLEMENTED RSCC

\begin{tabular}{|l|c|}
\hline Component & Volume \\
\hline 2 x Bus capacitors Ceralink 20uF & $17 \mathbf{~ c m}^{3}$ \\
\hline Resonant Film Capacitors 3uF & $\mathbf{7 \mathbf { c m } ^ { 3 }}$ \\
\hline $\begin{array}{l}\text { 55uH Resonant Inductance MPP } 55071 \\
\text { (30 turns, litz wire, 70 strands, } \varnothing=0.2 \mathrm{~mm} \text { ) }\end{array}$ & $\mathbf{3 0} \mathbf{~ c m 3}$ \\
\hline Transistors 2 GaN FETs per switch & $\mathbf{1 0} \mathbf{c m 3}$ \\
\hline Total & $\mathbf{6 4} \mathbf{~ c m 3}$ \\
\hline
\end{tabular}

is observable, especially in the case of low volume designs, when it is necessary to use a small inductance.

This effect leads to undesirably high switching losses if transistors with high $C_{\text {oss }}$ are employed, and the result is that the minimum volume is limited.

The theoretical analysis in [22] has shown that in the case of Si CoolMOS by changing the position of the resonant inductor it is possible to decrease the volume of the RSCC by $30 \%$ while the power losses could be, almost, halved for the same converter volume. However, the most important conclusion is that using the dc resonant converter it is possible to find solutions that are more efficient and smaller than any other RSCC implemented with an ac resonant inductor. On the other hand, if GaN FETs are used, there is not any drastic improvement in the converter's nominal efficiency or size between these two approaches, although the converter's efficiency will be dramatically improved just by changing the transistor technology, which is discussed later.

The most important difference between these two approaches can be seen at very small load or at no-load condition. For both semiconductor technologies, Si CoolMOS or GaN FET, the approach with dc resonant inductor will work in no-load conditions with the switching frequency very close to the resonant, while in the case of the ac resonant inductor this is not possible, as the switching frequency will be several times higher than the resonant one.

As possible candidates for the prototype design, solutions that have power losses up to $6 \mathrm{~W}$ and volumes less than $55 \mathrm{~cm}^{3}$ were selected. After more detailed thermal analysis of the resonant inductor, the design from Table II was selected. As it can be seen, the final resonant inductor was implemented with molypermalloy powder (MPP) magnetic material as it showed better results than a ferrite inductor of identical volume from the point of view of core losses and thermal stability. The resonant inductor implemented with MPP material will have decreased inductance at high current levels, which will produce a resonant response that will not be purely sinusoidal, but this does not cause any problem as it is shown later in experiments. For the bus capacitors, $C_{1}$ and $C_{2}$, CeraLink capacitors were selected due to their high power density. On the other hand, for the resonant capacitor multilayer ceramic capacitor (MCC), with C0G, X6S or X7T dielectrics, and film capacitors were analyzed. Nevertheless, after a series of experiments in which the resonant capacitor was stressed and the temperature rise was observed [3] X7T and film capacitors were chosen as possible resonant capacitor technologies. A series of experiments to characterize temperature rise of

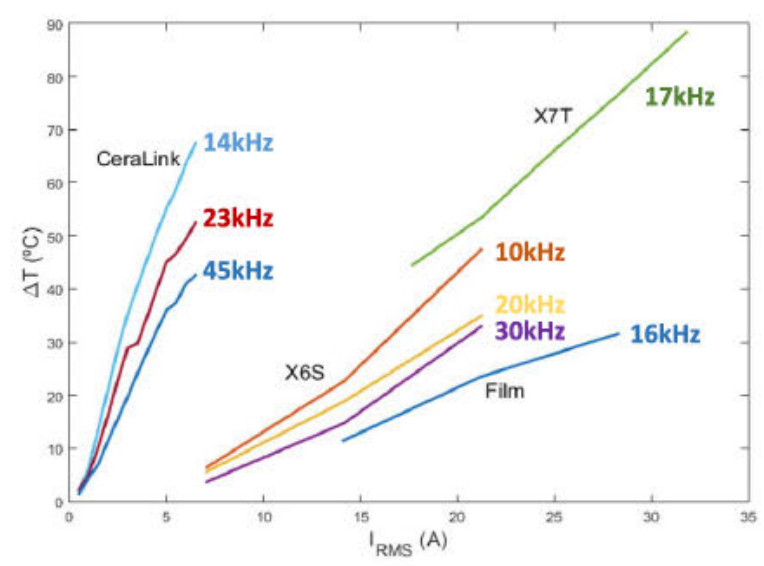

Fig. 12. Temperature rise of the resonant capacitors in the case of different capacitor technologies.

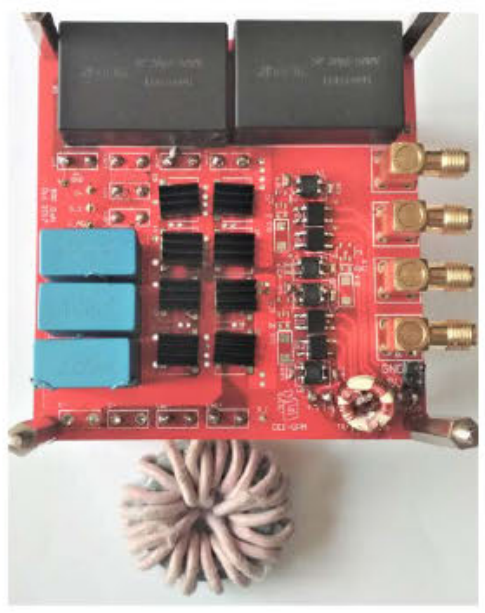

Fig. 13. Implemented RSCC.

the resonant capacitors was conducted. During the tests the capacitor technology was changed, maintaining the overall capacitance and the capacitors were stressed with different current amplitudes at different frequencies, as the equivalent ESR of the capacitor is frequency dependent. These tests helped us to identify the capacitor technology which would not decrease the converter robustness due to increased temperature. It is interesting to notice that film-based capacitors would provide better converter robustness as their temperature rise is significantly lower, but they occupy some more space than X7T MCC (see Fig. 12). Finally, in order to have a robust prototype, film capacitors were employed. CeraLink capacitors have the highest energy density but suffer from high ESR and, practically, were immediately discarded as a solution for the resonant capacitor.

The implemented prototype is capable of processing $4.5 \mathrm{~kW}$, as demanded by the specifications. Fig. 13 shows a photograph of the implemented converter and Table II details regarding the employed components.

\section{EXPERIMENTAL RESUlts AND ANALYSis OF THE RESONANT INDUCTOR CURRENT}

Figs. 14 and 15 show the most important waveforms of the analyzed converters, the resonant current, and drain-source 


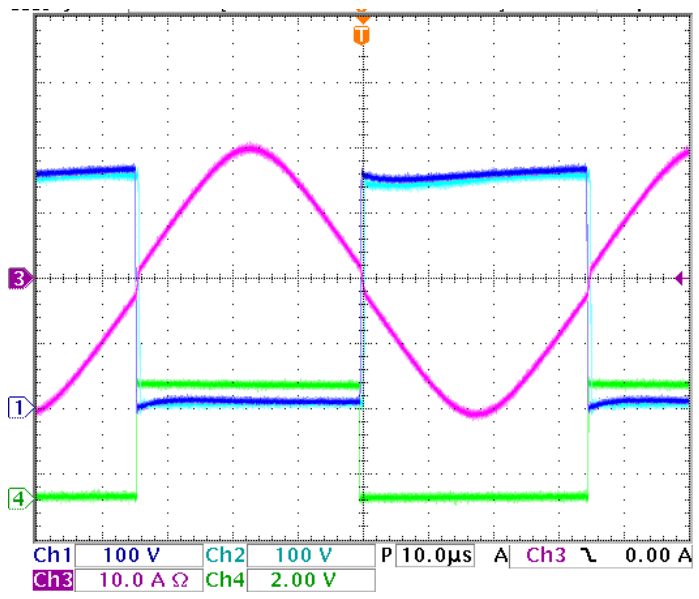

Fig. 14. Resonant current (pink curve), MOSFET drain-source voltage (blue line), and MOSFET gate-source voltage (green line) in the case of the ac resonant inductor at full-load power $(4,5 \mathrm{~kW})$.

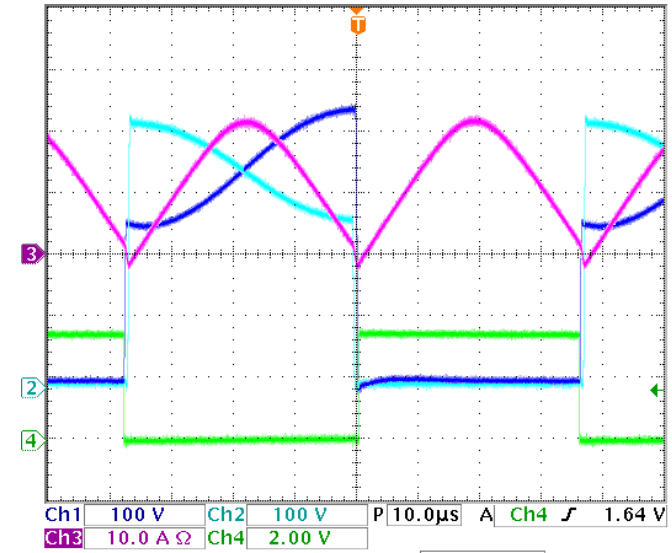

Fig. 15. Resonant current (pink curve), MOSFET drain-source voltages (blue and cyan lines), and MOSFET gate-source voltage (green line) in the case of the dc resonant inductor at full-load power (4 and $5 \mathrm{~kW})$.

voltage of $\mathrm{Q}_{2}$ and $\mathrm{Q}_{4}$, at full-load conditions, $V_{\text {out }}=700 \mathrm{~V}$ and $P_{\text {out }}=4.5 \mathrm{~kW}$. In both cases, the peak of the resonant current is around $20 \mathrm{~A}$, which concurs with the theoretical expectation $\left(I_{\text {peak }}=\pi I_{\text {out }}\right)$. The conducting FET is turned off with, approximately, $2 \mathrm{~A}$, and, as soon as it is turned off, the FET's parasitic capacitances start to resonate with the resonant inductor and the drain-source voltages start to charge/discharge. This transition can be observed as a fast linear current change in a very short period of time in Figs.14 and 15. As earlier explained, the conducting FET must be turned off with sufficient levels of resonant current due to $C_{\text {oss }}$ of the employed FET and its highly nonlinear characteristic. Another important detail that must be observed is the voltage that has to be sustained by FETs. In the case of the approach with the ac inductor, the FET voltage is clamped to the half of the output voltage. In the case of dc resonant inductor, this voltage is not clamped, and it has a high-voltage ripple that comes from the resonant capacitor voltage. As it can be observed, this voltage ripple can be as high as $150 \mathrm{~V}$ at full load and it can penalize the FET selection in the case of low resonant capacitor and high output power.

At nominal and low levels of the load (below $1.5 \mathrm{~kW}$ ), when the peak of resonant current is low, the resonant current does

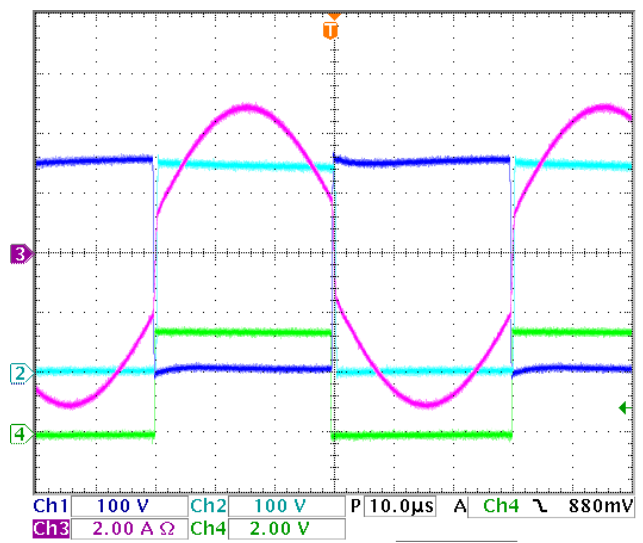

Fig. 16. Resonant current (pink curve), MOSFET drain-source voltage (blue and cyan lines), and MOSFET gate-source voltage (green line) in the case of the ac resonant inductor at $1-$ and $5-\mathrm{kW}$ load power.

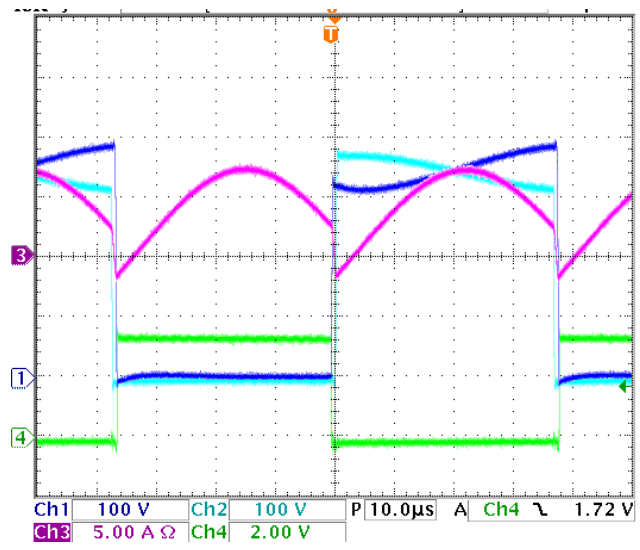

Fig. 17. Resonant current (pink curve), MOSFET drain-source voltages (blue and cyan lines), and MOSFET gate-source voltage (green line) in the case of the dc resonant inductor at $1-, 5-\mathrm{kW}$ load power.

not have a sinusoidal shape anymore as the turn-off current, needed for ZVS, is comparable with the peak value of the resonant current. For example, Figs. 16 and 17 show the most important waveforms in the case of the nominal load, $P_{\text {out }}=$ $1.5 \mathrm{~kW}$, for both analyzed approaches. In both experiments, the turn-off current is around $2 \mathrm{~A}$, but the difference between the switching period becomes obvious.

The switching frequency of the converter that uses the ac resonant inductor is $25 \%$ higher $(16 \mathrm{kHz}$ at $1.5 \mathrm{~kW}$ in comparison with $13 \mathrm{kHz}$ at $4.5 \mathrm{~kW}$ ) and the peak of the resonant current is lower than in the case of the resonant converter with the dc resonant inductor. The switching frequency is faster as the resonance starts at current level that is comparable with the peak of the resonant current and the level of turn-off ZVS current. In addition, as the resonant current has a partial sine wave shape, its mean value is higher than the mean value of the rectified sine wave and, consequently, the resonant current peak is lower than the theoretical expectation. If we continue to lower the load, the resonant current in the case of ac resonant inductor is even more distorted like it is shown in Fig. 18 in the case of a 500-W load. The resonant current has a trapezoidal shape, while the switching frequency is $160 \mathrm{kHz}$, which is more than ten times the resonant frequency. Clearly, such a high switching frequency at light load is not desirable due to 


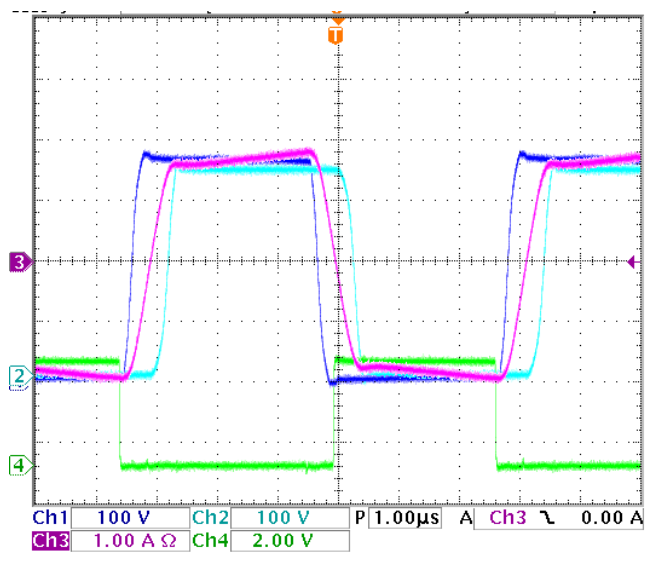

Fig. 18. Resonant current (pink curve), MOSFET drain-source voltage (blue and cyan curves), and MOSFET gate-source voltage (green line) in the case of the ac resonant inductor at $500-\mathrm{W}$ load power.

high switching losses as it produces significant losses in the magnetic core, conductors, and transistors. In order to avoid these losses, previously explained cycle skipping modulation should be applied in order to increase the peak of the resonant current as it will be presented later. If the load was further decreased, it would be impossible to reach ZVS conditions and, consequently, hard switching would occur. Moreover, the resonant converter would not operate with constant voltage gain of two. This same problem occurs if Si CoolMOFETs are employed, but at higher power levels as $I_{\mathrm{open}}$ and $I_{\text {close }}$ have higher values [22].

From the point of view of magnetic design and performance, if we compare the two analyzed approaches, the dc resonant inductor handles only one half of the excursion of the magnetic field but at the double frequency. As the $\beta$ factor in the Steinmetz equation is, approximately, between 2 and 2.5 for the materials of interest, the core losses in the worst case will be equal in both resonant converters (at the same switching frequency) as the $\alpha$ factor should be higher than 2 to have higher cores losses in the case of the dc resonant inductor. Speaking about the losses in the conductors, dc resonant inductor has a major part of the current in the dc part of the spectrum and, therefore, the conduction losses will be smaller. However, the biggest advantage of the RSCC with dc resonant inductor is that it can correctly operate even without load. To keep the voltage of the bus capacitors balanced, RSCC has to operate even without load. When the dc resonant inductor is used it does not need any special modulation, like cycle skipping, to operate correctly fulfilling ZVS conditions and keeping the voltages balanced.

\section{A. RSCC Operating at Low Loads}

As it has been explained earlier, the peak of the resonant current decreases when the output power is and in the case of the ac resonant inductor this might be a problem because as the peak of the resonant current falls below the value that is necessary to achieve ZVS could lead to important power losses.

Even more, in the case of the analyzed system, in the lowpower conditions this is what exactly happens and, in order

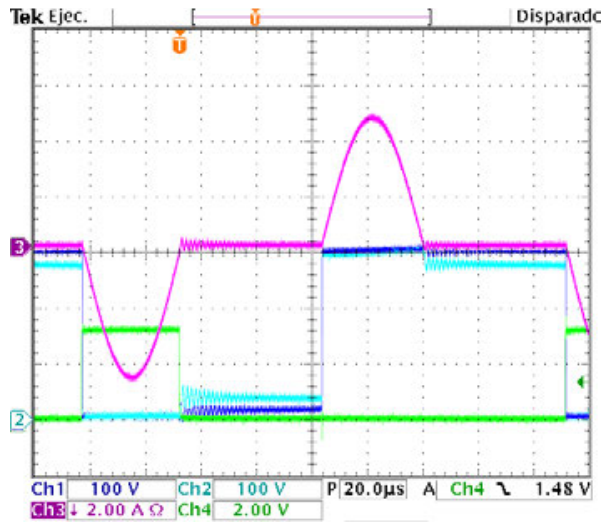

Fig. 19. Half-cycle resonance. Resonant current (pink trace), MOSFET drain-source voltages (blue and cyan traces), and gate-source voltage (green trace) in the case of ac resonant inductor at 500-W output power.

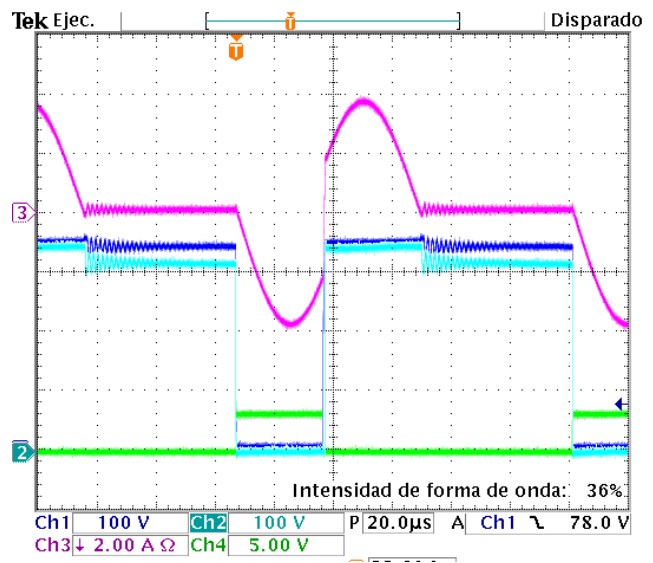

Fig. 20. Full-cycle resonance. Resonant current (pink trace), MOSFET drainsource voltages (blue and cyan traces), and gate-source voltage (green trace) in the case of ac resonant inductor at 500-W output power.

to avoid it, it is necessary to employ cycle skipping. For example, if one resonant cycle is skipped, the peak of the resonant current is doubled and ZVS could be achieved. Figs. 19 and 20 show the possible cycle skipping strategies. In the first strategy, the resonance is started, the energy from the input source is given to the resonant capacitor, and it is let to naturally end after half the resonant cycle by opening all the switches. The pause is made and then the second half of resonant cycle is performed in which the energy from the resonant capacitor is given toward the load and toward the high side bus capacitor. It can be seen that whenever the current reaches zero, and all the switched are left open, there are small high-frequency oscillations of drain-source voltage (blue trace) due to the resonance between the FET $C_{\text {oss }}$ and resonant inductor. This kind of oscillations can be observed in any converter that operates in the discontinuous conduction mode. These oscillations produce power losses which are not present during the normal operation. In addition, in order to start the resonance, hard discharge of the MOSFET's $C_{\text {oss }}$ occurs and soft turn-on is impossible. In order to decrease the amount of power losses due to these two mechanisms, in the second approach, a complete resonant cycle is performed, with one ZVS transition, and then the needed pause is made (see Fig. 20). With this strategy, the power losses due to hard 


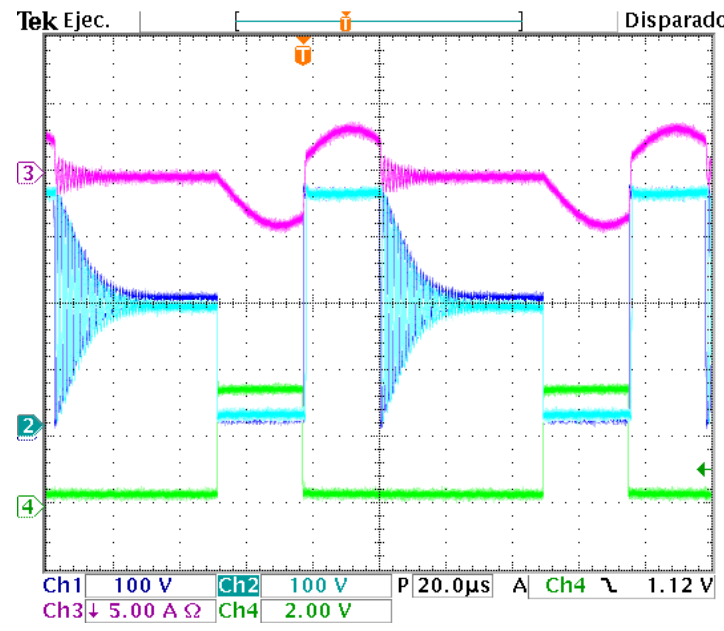

Fig. 21. High-frequency oscillations during one cycle resonance cycle skipping when the switches are not open correctly (resonant current was not equal to zero). Resonant current (pink trace) and MOSFET drain-source voltages (blue and cyan traces) in the case of ac resonant inductor at $500-\mathrm{W}$ output power.

turn-on and due to the parasitic oscillations are decreased at one half.

Figs. 19 and 20 show the experimental results for 500-W load, and it can be seen that the resonant current is a sine wave and that the switching frequency is below the resonant frequency, which are great benefits in comparison with results from Fig. 18. As the pause between two resonant cycles lasts, approximately the same as the resonant cycle, the amplitude of the resonant current is, approximately, doubled from the theoretical value. For the analyzed RSCC converter at $500 \mathrm{~W}$ at $V_{\text {out }}=700 \mathrm{~V}$, the peak of the resonant current is, approximately, $2 \pi I_{\text {out }}$, and the power losses were $2.5 \mathrm{~W}$ for the half-resonance cycle strategy and $2 \mathrm{~W}$ in the case of a full-resonance cycle approach. When the cycle skipping is applied, it is of the outmost importance to turn-off all the switches when there is no current in the resonant inductor to avoid high-frequency oscillations that produce additional losses and electromagnetic interference. Fig. 21 shows the huge voltage oscillations when the switches were left open at resonant current of $2 \mathrm{~A}$.

Nevertheless, due to very low $C_{\mathrm{oss}}$ of $\mathrm{GaN}$ transistors, it has been measured that the additional energy loss during these oscillations is below $1 \mathrm{~W}$, which would not be the case if CoolMOS were used, as it will be shown by the experimental results. In the case of the RSCC with dc resonant inductor the ZVS condition is easier to accomplish. When the ZVS transient is finished, the resonant current is negative and this starting point for the next resonance helps increasing the peak of the resonant current (in order to maintain the needed dc level of the input current). Even when the RSCC is without load, the current will resonate and the FET transitions are soft (see Fig. 22). Under no-load condition, the measured power losses were less than $2 \mathrm{~W}$.

\section{B. Power Loss Measurements and Comparison With Si CoolMOS}

Tables III and IV show the measured power losses and the measured temperature of the resonant inductor and of the

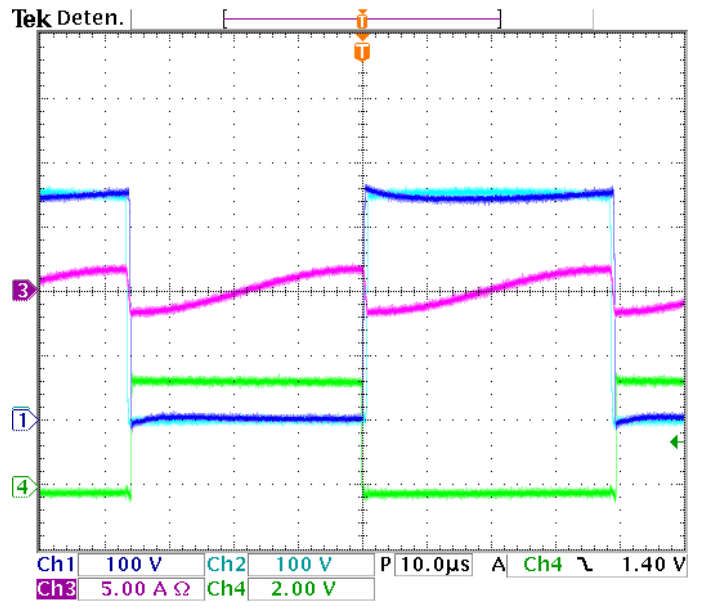

Fig. 22. Resonant current (pink trace), MOSFET drain-source voltages (blue and cyan traces), and MOSFET gate-source voltage (green trace) when RSCC operates without load-case of dc resonant inductor.

employed transistors for both resonant converters. Having in mind that power losses are very small, the precision of the power loss measurements is of crucial importance.

In the situations when the converter's efficiency is very high (higher than 99\%), the best way to measure power losses is using calorimetric measurements or to use "back-toback" approach. Unfortunately, both approaches are relatively complicated, and we opted to measure the dc voltages and currents with multimeter (Fluke 8808A) together with component temperatures. The MOSFETs were independently stressed with dc currents to obtain correlation between the package temperature and MOSFET power losses and, in that way, to double check measurements obtained with multimeters. The temperature measurement in dc conditions does not cover power losses in the resonant inductor magnetic core and in the employed resonant and bus capacitors. These losses were estimated using information from datasheet. CeraLink bus capacitors have ESR of, approximately, $16 \mathrm{~m} \Omega$, while MPP core loss was calculated using curves that have in mind nonlinear permeability of powder cores. The measured total power losses are contrasted with the estimated total power losses. As it can be seen from Tables III and IV, the estimation of the power losses confirms that the measured power losses are close to the estimated losses but at low output power level more precise measurement is needed.

In both resonant converters when the load was at $4.5 \mathrm{~kW}$, additional forced air cooling was applied as the inductor core temperature was too high due to high core and winding losses. At power levels below $500 \mathrm{~W}$, the operating frequency of the ac resonant inductor becomes, approximately, ten times higher $(160 \mathrm{kHz})$ than the resonant frequency and it affects heavily the overall efficiency, and, as earlier explained, at no-load condition, the situation is even worse. As it can be seen in Table III, estimated FET turn-off and inductor core losses are the main reason for the high overall losses, as they are heavily dependent on the frequency of operation. Increased power losses are additionally reflected in the measured component temperature. The core temperature is $30{ }^{\circ} \mathrm{C}$ higher in the case of ac resonant inductor, while the FET has $10{ }^{\circ} \mathrm{C}-15^{\circ} \mathrm{C}$ higher temperature. 
TABLE III

Measured Total Power Losses and Component Temperatures Together With Estimated Total Power Losses in the CASE OF RSCC With AC RESONANT INDUCTOR

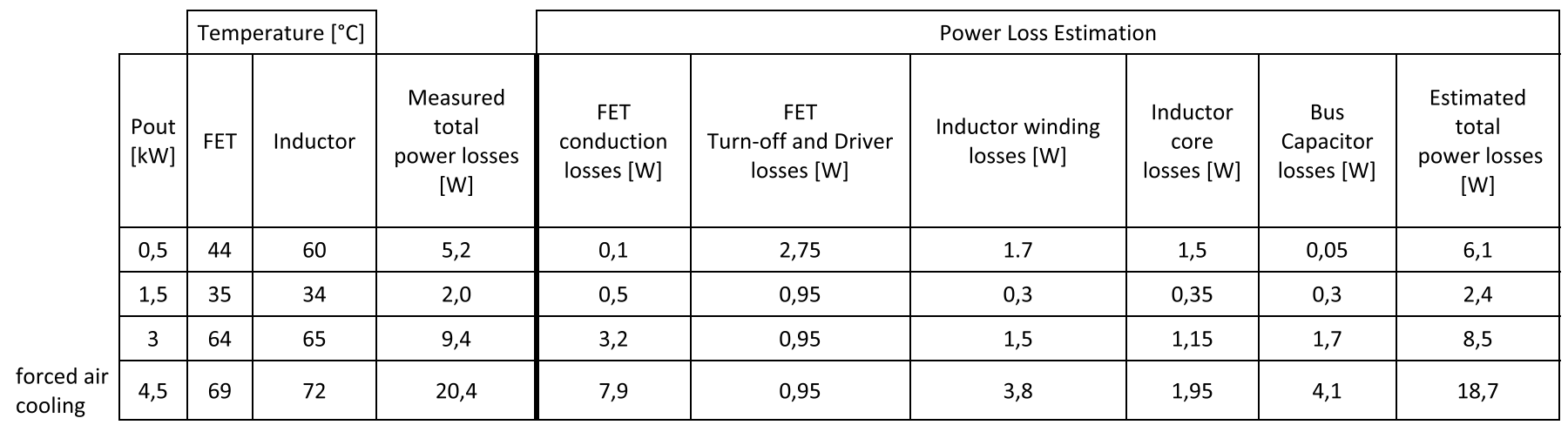

TABLE IV

Measured Total Power Losses and Component Temperatures Together With Estimated Total Power Losses in the CASE OF RSCC WITH DC RESONANT INDUCTOR

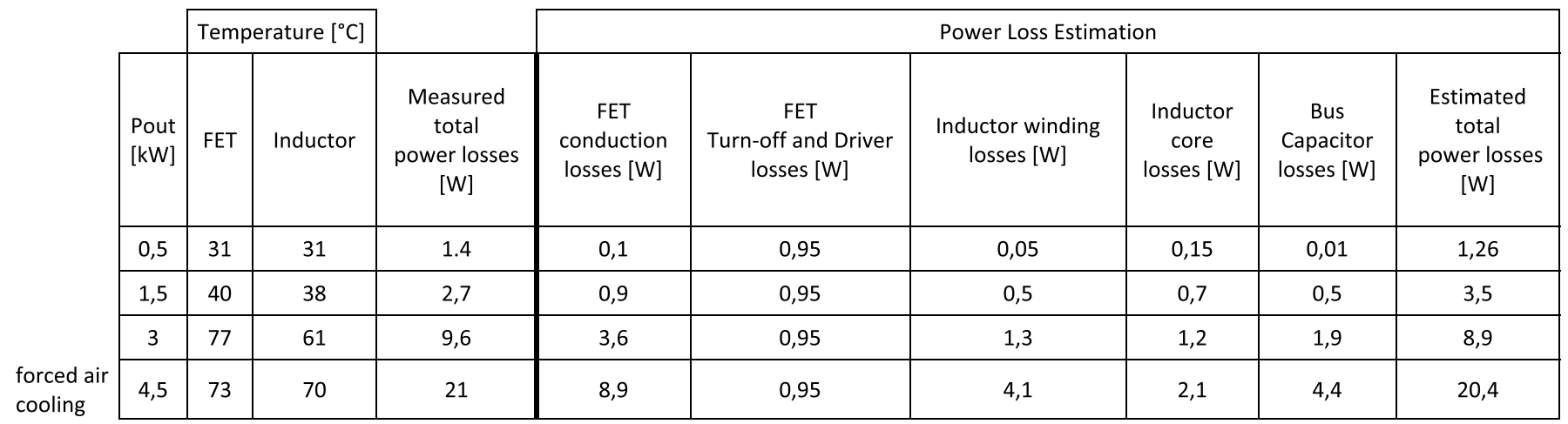

Due to the problem of increased switching frequency at low load, the explained cycle skipping is applied and the power losses are measured in these conditions. Applying cycle skipping at output power of $500 \mathrm{~W}$, the power loss of the resonant converter with ac inductor can be halved, but it is still slightly higher than in the case of the resonant converter with dc resonant inductor. On the other hand, the resonant converter with dc resonant inductor operates in a quite efficient way even at low power levels and no-load conditions. It must be stressed, once again, that in the case of the dc resonant inductor there is no need to perform any change in the modulation like in the case of the ac resonant inductor and that the cycle skipping is a direct consequence of the trasistor's $C_{\text {oss }}$. Fig. 23 shows an overview of the measured power losses for different output powers for both types of the resonant inductor.

In order to demonstrate advantages of the $\mathrm{GaN}$ technology, the same series of experiments was conducted using $\mathrm{Si}$ CoolMOS device, IPB65R045C7. First, the ZVS transients are compared (maintaining the rest of the components) using dc resonant circuit, and in Fig. 24, it can be seen that in the case of Si CoolMOS, we need more current in the resonant inductor at the beginning of the ZVS transition as expected to higher $C_{\text {oss }}$ of Si-based FETs (4.2 A for CoolMOS against $1.8 \mathrm{~A}$ for $\mathrm{GaN}$ FET). Another consequence of higher $C_{\mathrm{oss}}$ is a longer transient ( $2 \mu$ s for CoolMOS against $800 \mathrm{~ns}$ for GaN FET) that leads to a higher value of $I_{\text {close }}(-4.5 \mathrm{~A}$ for CoolMOS against -1.6 A for GaN FET). Higher $I_{\text {close }}$ provokes another

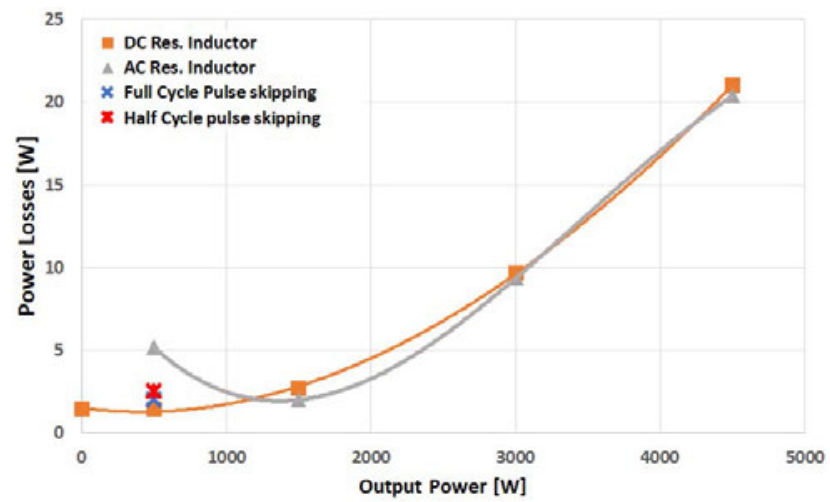

Fig. 23. Power losses of the presented converter in the case of ac and dc resonant inductor at different loads, and power losses when the half-cycle and full-cycle pulse skipping is applied at $500 \mathrm{~W}$ of output power.

effect; higher peak value of the resonant current as the average value must be the same for the same output current as it can be seen in Fig. 25 (25 A in the case of Si CoolMOS and $23 \mathrm{~A}$ in the case of GaN FETs).

Overall, in the analyzed circuit and for the same load current, the benefits of GaN FET over Si CoolMOS are as follows.

1) The rms value of the resonant current is $8 \%$ higher in the case of the converter based on Si CoolMOS

2) The $I_{\text {open }}$ is more than two times higher in the case of Si CoolMOS provoking higher turn-off losses 


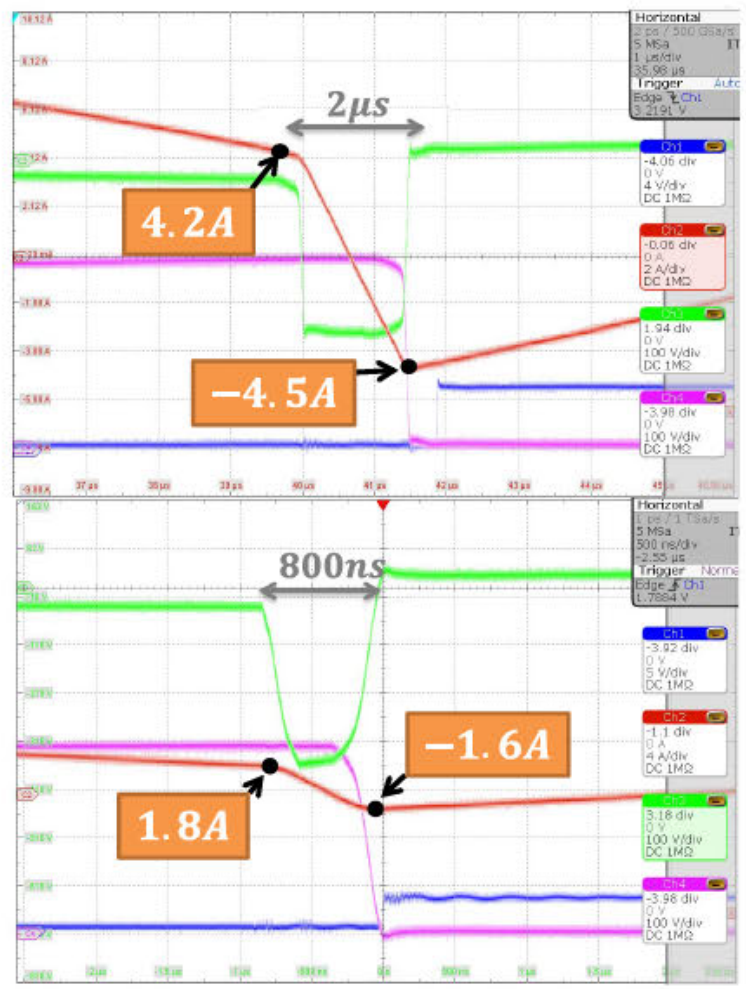

Fig. 24. ZVS transition in the case of Si CoolMOS device (left) and in the case of GaN FET device (right). Resonant current (orange trace), resonant inductor voltage (green trace), drain-source voltage (pink trace), and gate voltage (blue trace) are shown.

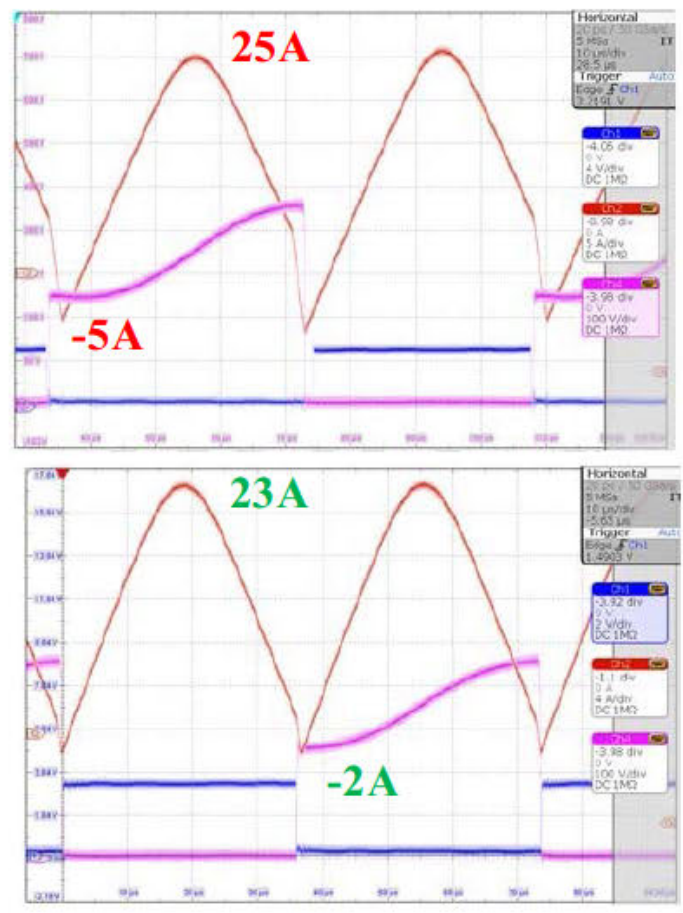

Fig. 25. Current of the resonant inductor at full load in the case of $\mathrm{Si}$ CoolMOS device (left) and in the case of GaN FET device (right). Resonant current (orange trace), resonant capacitor voltage (pink trace), and gate voltage (blue trace) are shown.

3) Partial ZVS transition is necessary in the case of $\mathrm{Si}$ CoolMOS and that generates additional switching losses It can be concluded that simply changing the transistor, the conduction losses should decrease around $40 \%$ (resistance

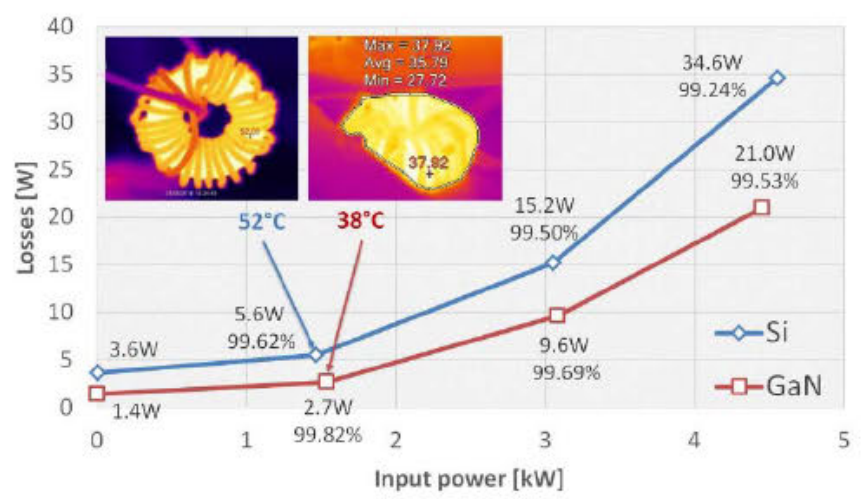

Fig. 26. Measured power losses in the case of dc resonant circuit implemented with Si CoolMOS and GaN FETs.

decreases $30 \%$ and $I_{\mathrm{rms}} 8 \%$ ) in the case of the maximal load $\left(P_{\text {out }}=4.5 \mathrm{~kW}\right)$. Fig. 26 presents the measured power losses for both technologies where the GaN advantage is shown for the complete load range. Important power savings can be seen even at low power levels. It is important to notice the influence of the higher rms current on the inductor temperature at nominal load in the case of Si-based converter $\left(52{ }^{\circ} \mathrm{C}\right.$ in the case of $\mathrm{Si}$ design against $38^{\circ} \mathrm{C}$ in the case of $\mathrm{GaN}$ design).

It is interesting to notice that the difference between the $\mathrm{dc}$ and ac resonant circuit in the case of Si CoolMOS is significantly higher due to higher $C_{\text {oss }}$ of device [22]. When the converter is implemented with $\mathrm{Si}$ CoolMOS, the switching frequency is three times higher than the resonant frequency at load level of $1,5 \mathrm{~kW}$. This provokes high losses, and heavily influences the optimization process and penalizes the size of the converter based on the ac resonant inductor. These losses can be alleviated using pulse skipping (producing $12 \mathrm{~W}$ of losses using full-wave pulse skipping), but they are still significantly higher than the losses in the case of the dc resonant inductor at the same load. On the other hand, $\mathrm{GaN}$ transistors have such a small $C_{\text {oss }}$ that its influence has been seen at load levels below $1 \mathrm{~kW}$, while at higher power levels there is not a clear conclusion which approach is better, for the analyzed specifications of the converter.

\section{CONCLUSION}

Multistage converters that include an RSCC are normally employed in the solutions that require high efficiency and power density. We have presented a design of a voltage doubler based on an RSCC that can process up to $4.5 \mathrm{~kW}$ and has high power efficiency (close to $99.5 \%$ ) and reach high power densities. Such a high-power efficiency is obtained thanks to GaN FETs that were used and to ZVS transitions that were used in full output power range.

It has been demonstrated that the ZVS performance of the analyzed converter is heavily influenced with the transistor technology. GaN FETs have significantly lower $C_{\text {oss }}$ than $\mathrm{Si}$ CoolMOS, and full ZVS transition can be achieved easily, while in the case of $\mathrm{Si}$ MOSFET only a partial ZVS can be achieved. Full ZVS transitions in combination with low channel resistance of 
GaN FETs lead to a solution that has decreased total power losses by $30 \%-50 \%$ just by changing the semiconductor technology.

Two possible implementations of the voltage doubler were analyzed and discussed. The voltage doubler can be implemented with the resonant inductor in series with the resonant capacitor (ac inductor approach) or with the resonant inductor in series with the input source (dc inductor approach). The main difference between these two implementations is in the shape and frequency of the resonant current. In the case of the ac resonant inductor, the shape and frequency of the current are load dependent. It is a sine wave, for heavy loads, while in the case of light loads, the current has, practically, trapezoidal waveform. In the case of the dc resonant inductor, the current is a rectified sine wave almost for all the load levels.

This difference in the current waveforms is of the outmost importance when the analyzed voltage doubler operates at light load and full load. At light load, the switching frequency of the voltage doubler with ac resonant inductor becomes several times higher (in the analyzed case, it was ten times higher) than the resonant and switching losses start to dominate the total power losses.

In order to alleviate the operating conditions at light loads, RSCC with ac resonant inductor must be combined with cycle skipping technique that helps to half the power losses. On the other hand, RSCC with dc resonant inductor operates at all load conditions without any problem, obtaining full ZVS transitions. For the analyzed specifications, there is not a clear conclusion which approach is better (ac or dc resonant inductor) at higher load levels, as the measured power losses are similar. The reason for this is mainly due to high performance of GaN FETs (low channel resistance, low $C_{\mathrm{oss}}$, and low switching losses), and the advantages of the RSCC voltage doubler with dc resonant inductor are not as clear as in the case when the converter is implemented with Si CoolMOS. Nevertheless, the implementation with dc resonant inductor still offers a design that is functional at all load levels. More precise power loss measurements using calorimetric approach must be conducted for better characterization of the implemented converter, especially at low load levels.

\section{REFERENCES}

[1] M. Chen, K. K. Afridi, S. Chakraborty, and D. J. Perreault, "Multitrack power conversion architecture," IEEE Trans. Power Electron., vol. 32, no. 1, pp. 325-340, Jan. 2017.

[2] Y. Lei, W. C. Liu, R. Carl, and N. Pilawa-Podgurski, "An analytical method to evaluate and design hybrid switched-capacitor and multilevel converters," IEEE Trans. Power Electron., vol. 33, no. 3, pp. 2227-2240, Mar. 2018. doi: 10.1109/TPEL.2017.2690324.

[3] M. Vasic, J. A. Oliver, P. Alou, J. A. Cobos, and P. Grbovic, "Experimental evaluation of capacitors for high power resonant converters," in Proc. IEEE Int. Exhib. Conf. Power Electron. Int. Motion Renew. Energy Manage., Nuremberg, Germany, May 2017, pp. 1-6.

[4] I. Oota, T. Inoue, and F. Ueno, "A realization of low-power supplies using switched-capacitor transformers and its analysis," Electron. Commun. Jpn., vol. 66-C, no. 8, 1983.

[5] A. Ioinovici, "Switched-capacitor power electronics circuits," IEEE Circuits Syst. Mag., vol. 1, no. 3, pp. 37-42, 3rd Quart., 2001.
[6] F. Zhang, L. Du, F. Z. Peng, and Z. Qian, "A new design method for high-power high-efficiency switched-capacitor DC-DC converters," IEEE Trans. Power Electron., vol. 23, no. 2, pp. 832-840, Mar. 2008.

[7] M. Shen, F. Z. Peng, and L. M. Tolbert, "Multilevel DC-DC power conversion system with multiple dc sources," IEEE Trans. Power Electron., vol. 23, no. 1, pp. 420-426, Jan. 2008.

[8] O. Keiser, P. K. Steimer, and J. W. Kolar, "High power resonant Switched-Capacitor step-down converter," in Proc. IEEE PESC, Jun. 2008, pp. 2772-2777.

[9] D. Cao and F. Z. Peng, "Zero-current-switching multilevel modular switched-capacitor DC-DC converter," in Proc. IEEE ECCE, Sep. 2009, pp. 3516-3522.

[10] D. Cao and F. Z. Peng, "A family of zero current switching switchedcapacitor DC-DC converters," in Proc. IEEE APEC, Feb. 2010, pp. $1365-1372$

[11] M. Xu, J. Sun, and F. C. Lee, "Voltage divider and its application in the two-stage power architecture," in Proc. 21st Annu. IEEE Appl. Power Electron. Conf. Expo. (APEC), Mar. 2006, p. 7.

[12] Q. M. Jin and M. Vasic, "Optimized design of GaN switched-capacitorconverter-based envelope tracker for satellite application," IEEE Trans. Emerg. Sel. Topics Power Electron., vol. 5, no. 3, pp. 1346-1355, Sep. 2017.

[13] P. J. Grbovic, A. Lidozzi, L. Solero and F. Crescimbini, "Five-level unidirectional T-rectifier for high-speed gen-set applications," IEEE Trans. Ind. Appl., vol. 52, no. 2, pp. 1642-1651, Mar./Apr. 2016. doi: 10.1109/TIA.2015.2504469.

[14] K. Sano and H. Fujita, "Performance of a high-efficiency switchedcapacitor-based resonant converter with phase-shift control," IEEE Trans. Power Electron., vol. 26, no. 2, pp. 344-354, Feb. 2011.

[15] P. J. Grbovic, P. Delarue, and P. L. Moigne, "A novel three-phase diode boost rectifier using hybrid half-DC-bus-voltage rated boost converter," IEEE Trans. Ind. Electron., vol. 58, no. 4, pp. 1316-1329, Apr. 2011

[16] C. Schaef and J. T. Stauth, "A 3-phase resonant switched capacitor converter delivering $7.7 \mathrm{~W}$ at $85 \%$ efficiency using $1.1 \mathrm{nH} \mathrm{PCB}$ trace inductors," IEEE J. Solid-State Circuits, vol. 50, pp. 2861-2869, Dec. 2015.

[17] C. Schaef, E. Din, and J. T. Stauth, "A hybrid switched-capacitor battery management IC with embedded diagnostics for series-stacked li-ion arrays," IEEE J. Solid-State Circuits, vol. 52, pp. 3142-3154, Dec. 2017.

[18] E. Hamo, A. Cervera, and M. M. Peretz, "Multiple conversion ratio resonant switched-capacitor converter with active zero current detection," IEEE Trans. Power Electron., vol. 30, no. 4, pp. 2073-2083, Apr. 2015.

[19] E. Hamo, M. Evzelman, and M. M. Peretz, "Modeling and analysis of resonant switched-capacitor converters with free-wheeling zcs," IEEE Trans. Power Electron., vol. 30, no. 9, pp. 4952-4959, Sep. 2015.

[20] CoolMOS C7 650VCoolMOS C7 Power Transistor IPB65R045C7, Datasheet, Revision 2.1, document, 2013. [Online]. Available: https://www.infineon.com/dgdl/Infineon-IPB65R045C7-DS-v02_01en.pdf?fileId=db3a30433e78ea82013e790478550043

[21] GS66516T Top-Side Cooled 650 V E-Mode GaN Transistor Preliminary Datasheet, Revision 1804224, document. [Online]. Available: https://gansystems.com/wp-content/uploads/2018/04/GS66516T-DSRev-180422.pdf

[22] M. Vasic, D. Serrano, P. Alou, J. A. Oliver, P. Grbovic, and J. A. Cobos, "Comparative analysis of two compact and highly efficient resonant switched capacitor converters," in Proc. IEEE Appl. Power Electron. Conf. Expo. (APEC), San Antonio, TX, USA: Mar. 2018, pp. 2168-2174.

[23] D. Graovac, M. Pürschel, and A. Kiep, "MOSFET power losses calculation using the data-sheet parameters," Automot. Power, Infineon, Neubiberg, Germany, Appl. Note Version 1.1., 2006.

[24] K. Venkatachalam, C. R. Sullivan, T. Abdallah, and H. Tacca, "Accurate prediction of ferrite core loss with nonsinusoidal waveforms using only steinmetz parameters," in Proc. IEEE Workshop Comput. Power Electron., Jun. 2002. pp. 36-41.

[25] Core Loss Calculator. Accessed: Nov. 2018. [Online]. Available: www.engineering.dartmouth.edu

[26] X. Tang and C. R. Sullivan, "Optimization of stranded-wire windings and comparison with litz wire on the basis of cost and loss," in Proc. IEEE 35th Аnnu. Power Electron. Specialists Conf., Jun. 2004, pp. 854-860. 
[27] C. R. Sullivan, "Optimal choice for number of strands in a litzwire transformer winding," IEEE Trans. Power Electron., vol. 14, pp. 283-291, Mar. 1999.

[28] Software for Magnetics Design: LitzOpt. Accessed: Nov. 2018. [Online]. Available: http://www.engineering.dartmouth.edu

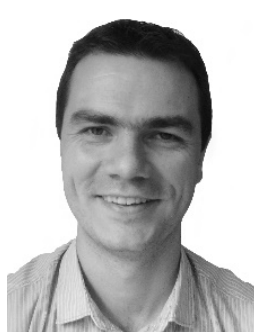

Miroslav Vasić (M'10) was born in Serbia, in 1981 $\mathrm{He}$ received the B.E. degree from the University of Belgrade, Belgrade, Serbia, in 2005, and the M.S. and Ph.D. degrees from the Universidad Politecnica de Madrid (UPM), Madrid, Spain, in 2007 and 2010, respectively.

He is currently an Assistant Professor with UPM. He has authored or co-authored over 50 papers in IEEE journals and conferences. He advised two $\mathrm{Ph} . \mathrm{D}$. theses and holds two patents. His current research interests include dc-dc converters, power converters for RF applications, and optimizations of converter topologies.

Dr. Vasić received the SEMIKRON Innovation Award for the team work on RF Power Amplifier with Increased Efficiency and Bandwidth, in 2012, a medal from Spanish Royal Academy of Engineering for his research trajectory as a Young Researcher in 2015, and the Best Young Researcher Award from the Universidad Politecnica de Madrid in 2016. He has been cooperating with the IEEE and other professional associations as a reviewer and a Session Chair.

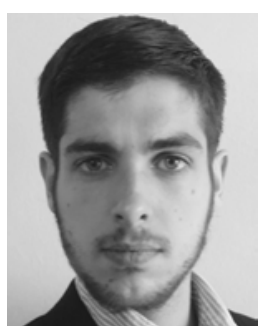

Diego Serrano received the B.S. degree in industrial electronic engineering from the University of Granada, Granada, Spain, in 2015, and the master's degree in electronic engineering from the Technical University of Madrid, Madrid, Spain, in 2016, where he is currently pursuing the Ph.D. degree.

His current research interests include dc and ac conversions for photovoltaic applications and multilevel converters.

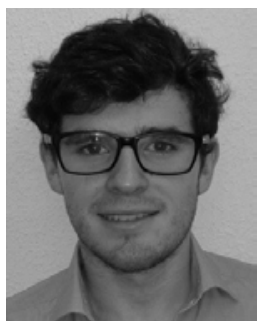

Victor Toral was born in Granada, Spain, in 1995. $\mathrm{He}$ received the B.Eng. degree, with valedictorian mention, in electronics engineering from the University of Granada, Granada, in 2017, and the M.Eng. degree in electronics engineering from the Polythecnic University of Madrid, Madrid, Spain, in 2018.

His current research interests include biomedical instrumentation and power electronics.

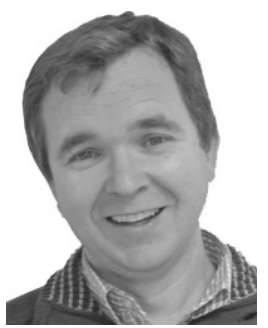

Pedro Alou (M'07) was born in Madrid, Spain, in 1970. He received the M.S. and Ph.D. degrees in electrical engineering from the Universidad Politécnica de Madrid (UPM), Madrid, in 1995 and 2004, respectively.

He has been involved in power electronics since 1995, participating in more than 50 research and development projects with the industry. He is currently an Associate Professor with UPM. He has authored or co-authored over 100 technical papers and holds five patents. His current research interests include power supply systems, advanced topologies for efficient energy conversion, modeling of power electronics, advanced control techniques for high dynamic response, energy management, and new semiconductor technologies for power electronics. His research activity is distributed among industrial, aerospace, and military projects.

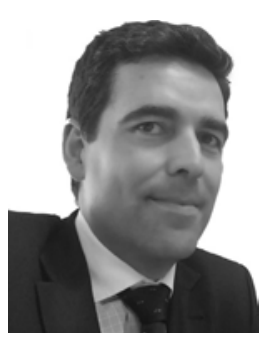

Jesus A. Oliver (M'07) received the master's and Doctoral degrees in electrical engineering from the Universidad Politécnica de Madrid (UPM), Madrid, Spain, in 1996 and 2007, respectively.

In 2001, he joined UPM as an Assistant Professor, where he became an Associate Professor in 2007. He has authored or co-authored over 150 scientific papers on journals and conferences. He holds five patents. His current research interests include modeling (dc-dc converters, magnetic components, piezoelectric transformers, fuel cells, and dc distributed power electronic systems), fast control techniques for dc-dc converters for voltage regulation module applications and RF amplifiers, three-phase rectifiers for aircraft applications, wireless power transfer, and power systems on chip.

Dr. Oliver has led numerous research projects with private and public funding. He has participated in more than 50 direct research and development projects with companies in Europe, USA, Australia, and China. He is currently serving as an Associate Editor for the IEEE TRANSACTIONS ON POWER ELECTRONICS.

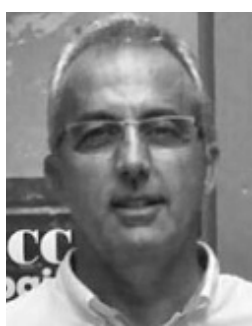

Jose A. Cobos (M'92-SM'12) was the Founder Director of the Centro de Electroìnica Industrial, Universidad Politécnica de Madrid (CEI-UPM), in 2006. He was an RCC Fellow with Harvard University, Cambridge, MA, USA, where he was a Fulbrighter with the University of California at Berkeley, Berkeley, CA, USA, from 2016 to 2017. Since 2016, he has been the Founder President of Industrial Council with CEI, to coordinate education and research with industry. $\mathrm{He}$ is currently a Full Professor with UPM. His contributions are focused on power supply systems for industrial, aerospace, telecom, automotive, renewable energy, and medical applications. He advised over 50 graduate students. He has authored or co-authored over 300 technical papers $(>8000$ citations, $h=47)$. He is the Co-Inventor of patents with six companies. His current research interests include energy efficiency in digital systems, RF amplifiers, renewable energy, magnetic components, transcutaneous energy transfer, and biomedical applications.

Dr. Cobos was an Adcom Member of the IEEE Power Electronics Society (IEEE-PELS) and the Chair of its Technical Committee on DC Power Supply Systems. He was the General Chair of PwrSoC 2016 (IEEE-PELS and Power Supply Manufacturers Association) and an Associate Editor of the IEEE TRAnsactions on Power Electronics and PELS Letters. He conducted professional seminars and tutorials in USA, U.K., Austria, Germany, Italy, Sweden, Switzerland, Syria, Mexico, and Macedonia. He is a Steering Committee Member (Program Chair in 2019) of the IEEE Applied Power Electronics Conference. 